\title{
Hemorrhagic Onset of Hemangioblastoma Located in the Dorsal Medulla Oblongata Presenting with Tako-Tsubo Cardiomyopathy and Neurogenic Pulmonary Edema: A Case Report
}

\author{
Masayuki Gekka Shigeru Yamaguchi Ken Kazumata \\ Hiroyuki Kobayashi Hiroaki Motegi Shunsuke Terasaka \\ Kiyohiro Houkin
}

Department of Neurosurgery, Graduate School of Medicine, Hokkaido University, Sapporo, Japan

\section{Key Words}

Baroreflex failure $\cdot$ Hemangioblastoma $\cdot$ Neurogenic pulmonary edema - Medulla oblongata Solitary tract nucleus - Tako-tsubo cardiomyopathy

\begin{abstract}
Here, we present a case of dorsal medulla oblongata hemangioblastoma with fourth ventricular hemorrhage. A 23-year-old female developed sudden consciousness disturbance, and $\mathrm{CT}$ revealed hemorrhage in all cerebral ventricles and a hyperdense mass in the cisterna magna. Although the reddish tumor located in the dorsal medulla oblongata was successfully removed, she suffered from severe tako-tsubo cardiomyopathy (TTC) and neurogenic pulmonary edema (NPE) because of baroreflex failure and damage to the solitary tract nuclei. After intensive care for 12 weeks following surgery, she was discharged without any neurological or radiological deficits. Pathogenesis of TTC/NPE is discussed in this paper.
\end{abstract}

(c) 2014 S. Karger AG, Basel

Shunsuke Terasaka, MD

Department of Neurosurgery, Graduate School of Medicine

Hokkaido University

N-14, W-7 Kita-ku, Sapporo 060-8638 (Japan)

E-Mail terasas@med.hokudai.ac.jp 
Gekka et al.: Hemorrhagic Onset of HB Located in the Dorsal Medulla Oblongata Presenting with TTC and NPE: A Case Report

\section{Introduction}

Hemangioblastoma (HB) is a benign tumor (WHO grade 1) that comprises $2 \%$ of all intracranial tumors [1]. The risk of spontaneous hemorrhage was reported by Gläsker et al. [2] as having a very low probability $(0.0024 \%$ per patient per year), and the majority of hemorrhagic events are subarachnoid and intracerebral bleedings [1]. Particularly, dorsal medulla oblongata $\mathrm{HB}$ with fourth ventricular hemorrhage is quite rare, and only 2 cases have been previously reported $[1,3]$.

The brainstem plays an important role in mediating the autonomic nervous system. Baroreflex failure is a neurovisceral disorder that selectively affects the network from the carotid sinus and aortic arch to the solitary tract nuclei, which regulates blood pressure and heart rate variability. Baroreceptors located in each carotid sinus, the aortic arch, and the great vessels of the thorax send information about distention of the vessel wall via the glossopharyngeal and vagal nerves to the solitary tract nuclei in the brainstem. In addition, the blood volume in the thorax is sensed by low pressure receptors linked by the vagal nerves to the solitary tract nuclei. Therefore, brainstem damage in the region of the solitary tract nuclei may result in baroreflex failure. This entails the loss of buffering of blood pressure and sympathetic activity, and is reported to be related to tako-tsubo cardiomyopathy (TTC) [4].

Here, we report a case of dorsal medulla oblongata HB presenting with fourth ventricular hemorrhage in a 23-year-old woman with no history of trauma or Von Hippel-Lindau (VHL) disease. Severe neurogenic pulmonary edema (NPE) and TTC occurred in the perioperative period. We present the clinical course of this rare case in detail and discuss some pathophysiological issues derived from this observation.

\section{Case Report}

Present Illness

A 23-year-old woman with no medical antecedents was admitted to our service because of a sudden headache, vomiting, and disturbance of consciousness while she was working.

\section{Examination}

Initial physical examination revealed disturbance of consciousness (Glasgow Coma Scale E3V4M5), without any deficits of brain stem function. A cranial CT revealed hemorrhage in all cerebral ventricles, acute hydrocephalus, and a hyperdense mass in the cisterna magna (fig. 1). Emergent angiography revealed tumor staining and several feeding vessels originating from the left posterior inferior cerebellar artery. According to these radiological findings, HB presenting as intraventricular hemorrhage was presumed as a preoperative diagnosis. Because her consciousness rapidly became worse (Glasgow Coma Scale E1V2M3), she underwent emergency surgical intervention immediately after an angiography.

\section{Surgical Procedure}

An urgent surgical removal of the tumor and external ventricular drainage were performed. The patient was placed in the prone position. After suboccipital midline incision, suboccipital craniotomy was performed. The reddish tumor measuring $3 \times 2.5 \times 2.5 \mathrm{~cm}$ was located in the dorsal medulla oblongata. The tumor feeders were carefully selected and sectioned, avoiding injury of pial passing arteries, and finally the tumor was removed in en 
Gekka et al.: Hemorrhagic Onset of HB Located in the Dorsal Medulla Oblongata Presenting with TTC and NPE: A Case Report

bloc fashion. During the operation, a large amount of secretions were suctioned through the endotracheal tube and refractory hypotension was recorded.

Pathology

The tumor was composed of a large number of microvascular dilated vessels and a rich population of stromal cells. The nuclei were hyperchromatic, but no prominent pleomorphism, nucleoli, or other atypical features were evident. Histologically, the tumor was diagnosed as HB.

Postoperative Course (fig. 2)

After surgery, the patient was treated in the intensive care unit because of cardiovascular/pulmonary system dysfunction. She woke up from anesthesia $12 \mathrm{~h}$ after surgery with a complete recovery of consciousness and no motor deficit.

The patient showed severe cardiovascular/pulmonary system dysfunction immediately after surgery, and high-dose norepinephrine and dobutamine were administrated to maintain a systolic blood pressure. After surgery, rapid testing for cardiac troponin $\mathrm{T}$ was positive and the serum C-reactive protein value was $1.36 \mathrm{mg} / \mathrm{dl}$. An electrocardiogram showed ST-segment elevation in the high lateral leads and ST-segment depression in V3-V6. An echocardiogram showed global hypokinesia of the left ventricular wall with severe mitral insufficiency and an estimated ejection fraction of $24 \%$. The condition was thus taken to be suggestive of TTC. Three days after the operation, the brain natriuretic peptide (BNP) level was $2,098 \mathrm{pg} / \mathrm{ml}$ (normal range: $0.5-30 \mathrm{pg} / \mathrm{ml}$ ) and increased up to $3,333 \mathrm{pg} / \mathrm{ml}$ on postoperative day (POD) 9.

The patient required hANP and furosemide for the treatment of heart failure. X-ray after operation showed a typical finding of NPE. After continuing the positive pressure ventilation, she was extubated on POD 6. She required noninvasive positive pressure ventilation for 4 days after extubation. Left ventricular function and BNP level had gradually normalized, and NPE was cured on POD 40.

Postoperative neurological examination showed dysphagia and ataxia from disturbance of deep sensibility derived from brain stem damage. After rehabilitation for dysphagia, ataxia, orthostatic hypotension and aglutition, she returned to normal status 12 weeks after surgery. Postoperative MRI showed no residual tumor (fig. 3).

\section{VHL Screening}

She had no family history of VHL disease, and a gadolinium MRI study of the whole neuraxis ruled out any other HBs. Abdominal CT showed no mass in the kidney, and ophthalmological examination denied retinal hemangiomas.

\section{Discussion}

Spontaneous hemorrhage is a very uncommon event among central nervous system HB. With respect to the fourth ventricular hemorrhage, only 2 cases have been previously reported. Interestingly, one of them also presented TTC and NPE, as in our case. This fact suggests that medulla oblongata HB potentially induces TTC/NPE by its hemorrhage and provides us with an important clue for understanding the trigger mechanism of TTC and NPE.

NPE and TTC may share a common phenotype, but have different etiologies. Although TTC has been acknowledged by the American Heart Association and the American College of 
Cardiology as a form of reversible cardiomyopathy, its exact pathophysiology has been unclear. The most accepted theory is myocardial stunning caused by a catecholaminemediated mechanism. Recently, TTC has been reported as an important pathophysiology secondary to subarachnoid hemorrhage, ischemic stroke, epileptic seizure, and severe neurological injury [5]. NPE is defined as an acute pulmonary edema occurring shortly after various injuries to the central nervous system (stroke, intracranial hemorrhage, seizures, etc.). NPE also remains poorly understood because of its complex pathophysiology, but a close connection is presumed between NPE and sympathetic activity [6]. It has been reported that TTC and NPE occasionally occur simultaneously in subarachnoid hemorrhage, as in this case [7].

In our patient, $\mathrm{HB}$ arose from the dorsal medulla oblongata, in which solitary tract nuclei are located. Solitary tract nuclei take on the important role of baroreflex. Increases in sympathetic activity are restrained by baroreflexes, which are classic negative feedback loops. Damage to the solitary tract nuclei causes insufficiency in this negative feedback loop and results in baroreflex failure. Baroreflex failure entails the loss of regulation of the excess sympathetic activity characterized by volatility of blood pressure and heart rate, which could lead to TTC. Uncontrollable sympathetic activity may also cause NPE.

\section{Conclusion}

Here, we presented a case of dorsal medulla oblongata HB with fourth ventricular hemorrhage. The patient suffered from severe TTC and NPE in her perioperative period. Damage to the solitary tract nuclei would be presumed as a cause of this unusual pathophysiology. After remaining in intensive care for 12 weeks following surgery, she was discharged without any neurological or radiological deficits.

\section{References}

1 de San Pedro JR, Rodríguez FA, Níguez BF, Sánchez JF, López-Guerrero AL, Murcia MF: Massive hemorrhage in hemangioblastomas Literature review. Neurosurg Rev 2010;33:11-26.

12 Gläsker S, Van Velthoven V: Risk of hemorrhage in hemangioblastomas of the central nervous system. Neurosurgery 2005;57:71-76.

-3 Fujii H, Higashi S, Hashimoto M, Shouin K, Hayase H, Kimura M: Hemangioblastoma presenting with fourth ventricular bleeding. Neurol Med Chir (Tokyo) 1987;27:545-549.

-4 Berganzo K, Ciordia R, Gómez-Esteban JC, Tijero B, Agundez M: Tako-tsubo cardiomyopathy in a patient with bilateral lesions in the dorsal medulla. Clin Auton Res 2011;21:65-67.

5 Lassnig E, Weber T, Auer J, Nomeyer R, Eber B: Pheochromocytoma crisis presenting with shock and takotsubo-like cardiomyopathy. Int J Cardiol 2009;134:e138-e140.

-6 Meyer S, Lindinger A, Löffler G, Limbach HG, Shamdeen MG, Gottschling S: Mechanisms of disease/hypothesis: neurogenic left ventricular dysfunction and neurogenic pulmonary oedema. Wien Med Wochenschr 2009;159:342-345.

7 Inamasu J, Nakatsukasa M, Mayanagi K, Miyatake S, Sugimoto K, Hayashi T, Kato Y, Hirose Y: Subarachnoid hemorrhage complicated with neurogenic pulmonary edema and takotsubo-like cardiomyopathy. Neurol Med Chir (Tokyo) 2012;52:49-55. 
Gekka et al.: Hemorrhagic Onset of HB Located in the Dorsal Medulla Oblongata Presenting with TTC and NPE: A Case Report

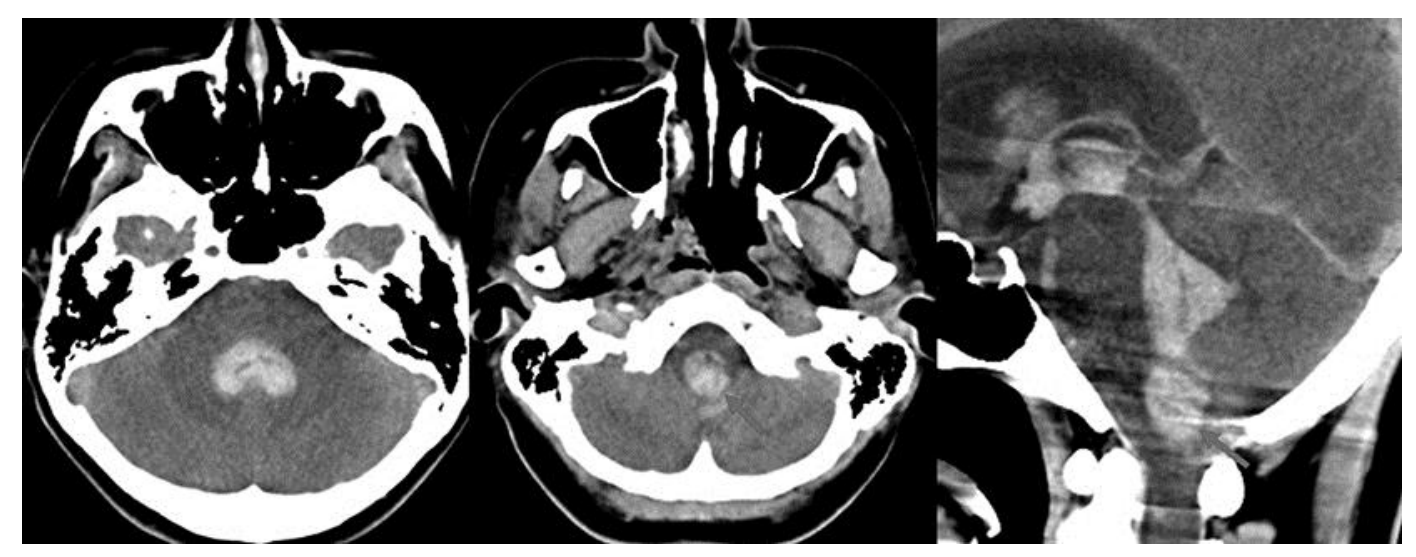

Fig. 1. Preoperative CT showed fourth ventricular hemorrhage and a hyperdense mass in the cisterna magna.

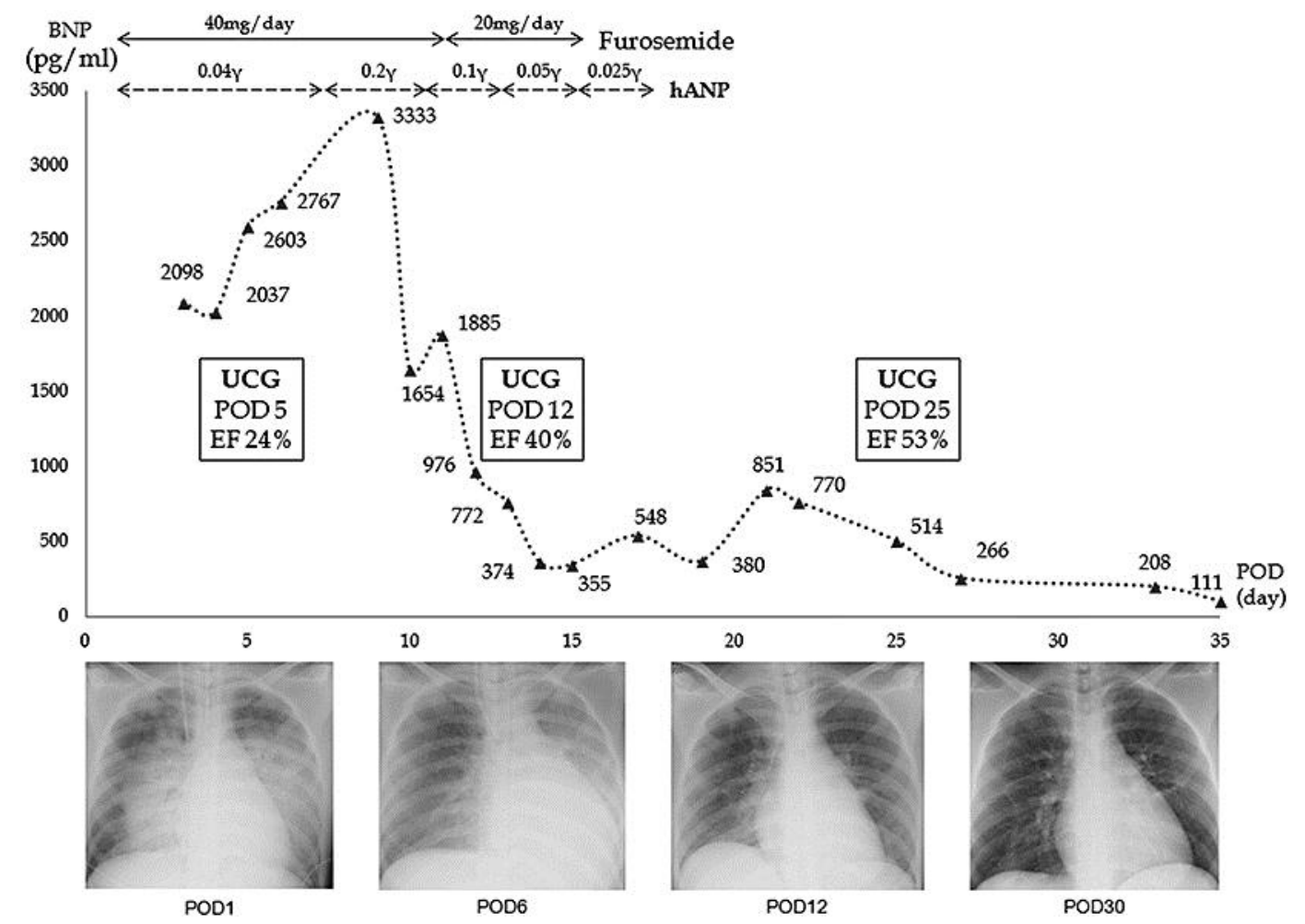

Fig. 2. Diagram of BNP level after surgery (graph) and serial chest X-ray findings. POD 1: large amount of bilateral pulmonary edema can be seen. POD 6: bilateral pulmonary edema and cardiac dilatation can be seen. POD 12: bilateral pulmonary edema is improved but sustained. POD 30: bilateral pulmonary edema has disappeared. Ultrasound cardiography (UCG) findings: on POD 5, the ejection fraction (EF) was $24 \%$. On POD 25, the EF was 53\%. 
Case Reports in
Neurology

Case Rep Neurol 2014;6:68-73

DOI: $10.1159 / 000361041$

(c) 2014 S. Karger AG, Basel

Gekka et al.: Hemorrhagic Onset of HB Located in the Dorsal Medulla Oblongata Presenting with TTC and NPE: A Case Report

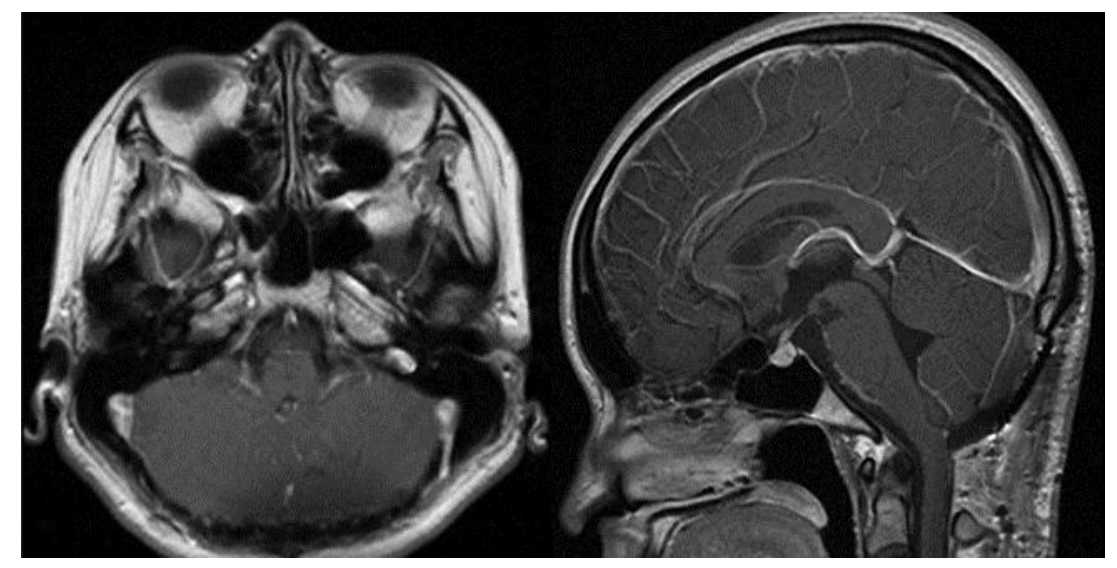

Fig. 3. Postoperative gadolinium-enhanced MRI showed no residual tumor. 